\title{
Polymorphic acetylation of sulphamethazine in a Zimbabwe population
}

\author{
CHARLES F B NHACHI \\ From the Department of Clinical Pharmacology, Godfrey Huggins School of Medicine, University of \\ Zimbabwe, Avondale, Harare, Zimbabwe.
}

SUMMARY Sulphamethazine, $8 \mathrm{mg} / \mathrm{kg}$ body weight, was administered orally in tablet form to 100 healthy volunteers and total and free sulphamethazine were determined in the six hour urine sample. The bimodal population frequency distribution for percentage acetylated sulphamethazine showed 42 of the tested population to be fast and 58 to be slow acetylators, that is, an estimation of $q=0.72 \pm 0.3$ as the frequency of the allele controlling slow acetylation. The study also revealed ample evidence that the assay of the drug in urine can be done in a significantly shorter time.

It has been established by a number of investigators that there is a genetic polymorphism in man for the acetylation of sulphmethazine. ${ }^{1-3}$ Subjects are generally classified as slow or rapid acetylators ${ }^{4-6}$ and the proportion of slow to rapid acetylators varies among different peoples, ${ }^{7}$ but still exhibits bimodality even among regional groupings of a population. ${ }^{89}$

The present study was carried out to establish the existence of genetic polymorphism in a Zimbabwean population. The study also presents evidence that the determination of the acetylator phenotype using the method of Bratton and Marshall as given by Varley ${ }^{10}$ can be achieved, even when the incubation time is reduced to 40 minutes and using a very small dose of sulphamethazine.

\section{Subjects, materials, and methods}

One hundred healthy volunteers (all native Zimbabweans) were employed in the study. The subjects, both male and female, were between the ages of 20 and 38 years and were drawn from the Medical School students, technicians, and hospital nurses.

Six hour urine samples were collected and the volume measured. Total and free sulphamethazine concentrations were determined, using the method of Varley, ${ }^{10}$ on the day of collection. The percentage

Received for publication 8 July 1986.

Revised version accepted for publication 14 November 1986. of sulphamethazine acetylated was determined using the formula

$\frac{\mathrm{T}-\mathrm{F}}{\mathrm{T}} \%$

where $\mathrm{T}=$ total sulphamethazine excreted after six hours, and $F=$ free (unacetylated) sulphamethazine excreted after six hours.

The percentage of sulphamethazine was also determined at different periods of acid hydrolysis in a boiling water bath for 10 subjects, who had been classified as slow (4) and fast (6) acetylators.

\section{Results}

The distribution of slow and fast acetylators of sulphamethazine in urine six hours after administration are shown in the figure and table 1. A

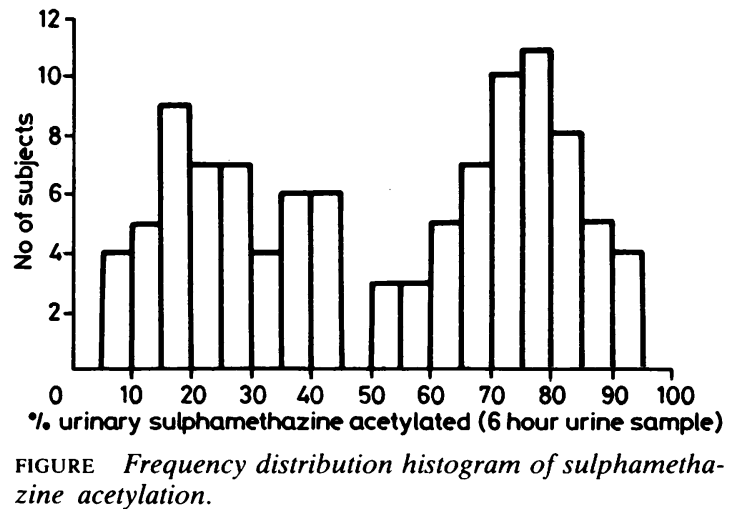


TABLE 1 Distribution of the acetylator phenotype among 100 subjects (70 men, 30 women).

\begin{tabular}{lcl}
\hline & Fast & Slow \\
\hline$\%$ acetylators & 42 & 58 \\
$\%$ women acetylators & 8 & 22 \\
$\%$ men acetylators & 34 & 36 \\
\hline
\end{tabular}

With one degree of freedom and $\alpha=0.05, \chi^{2}<3.841$, there is no significant difference in phenotype distribution between the sexes.

TABLE 2 Percentage of urinary sulphamethazine acetylated as determined after different periods of acid hydrolysis of urinary acetyl sulphamethazine.

\begin{tabular}{lccccccc}
\hline Subjects & \multicolumn{2}{l}{ Minutes } & \multicolumn{7}{l}{} \\
\cline { 2 - 8 } & 5 & 10 & 20 & 30 & 40 & 50 & 60 \\
\hline A & 4 & 4 & 18 & 27 & 39 & 47 & 47 \\
B & 86 & 88 & 92 & 93 & 94 & 94 & 95 \\
C & 33 & 48 & 57 & 63 & 63 & 68 & 68 \\
D & 10 & 15 & 20 & 28 & 38 & 42 & 45 \\
E & 70 & 77 & 81 & 84 & 86 & 87 & 88 \\
F & 60 & 63 & 65 & 65 & 69 & 70 & 70 \\
G & 37 & 63 & 65 & 65 & 69 & 70 & 70 \\
H & 9 & 15 & 39 & 52 & 53 & 53 & 55 \\
I & 39 & 57 & 63 & 70 & 71 & 72 & 74 \\
J & 40 & 66 & 63 & 74 & 74 & 74 & 75 \\
\hline
\end{tabular}

satisfactory separation (not completely resolved) of fast and slow acetylator phenotypes was obtained. (The phenotype is classified as slow if the percentage of acetylated sulphamethazine is less than $70 \%$ and fast if more than $70 \%$.)

As has been shown in previous studies, ${ }^{11}$ the urinary elimination of total sulphamethazine was significantly greater in fast acetylators (mean $5 \cdot 1$, 1.7 SEM) than in slow acetylators (mean 3.2, 1.6 SEM).

Table 2 shows that in the majority of cases and for practical purposes (qualitatively), the apparent percentage of urinary sulphamethazine acetylated changes very little, if at all, after 40 minutes of acid hydrolysis in a boiling water bath.

\section{Discussion}

Although the existence of a genetic polymorphism in man in general, and in an African population in particular, for the acetylation of sulphamethazine is well known, ${ }^{89}$ this study is the first to be done in Zimbabwe.

That the distribution of acetylation of sulphamethazine exhibits bimodality is confirmed by the results of the present study. However, as can be seen in the figure, there is an overlap. The six hour urine sample has been shown in other studies ${ }^{12}$ to give efficient discrimination between slow and fast acetylators. Analysis of a six hour plasma sample for percent acetylation would have given a bette resolution between phenotypes.

The method for determining the total amount sulphamethazine excreted in urine (based on the Bratton Marshall method as given by Varley ${ }^{10 \bar{x}}$ involves breaking down the acetyl derivative befor $\$$ the colour reaction. This is achieved by subjecting the urine sample to acid hydrolysis, that is, adding. hydrochloric acid and heating the sample in a boiling water bath for one hour. Although this method was used in the present study, it was shown that 4.6 minutes of hydrolysis is enough to give an efficien $\overline{\bar{P}}$ discrimination between slow and rapid acetylatorst as indicated in table 2 .

In remote centres of most developing countries where spectrophotometry is not automated, saving 20 minutes on every batch of assays (not more tha? 10 samples per batch) is a very important factor indeed.

Determination of the acetylator phenotype in Zimbabwean population was a worthwhile exercise in view of the fact that slow acetylators of certain. drugs, for example, isoniazid, are more susceptible to developing peripheral neuropathy than rapiæ̊ acetylators of the drug. ${ }^{13}$

Among other things, the present study provides evidence for the determination of the acetylato phenotype using a very low dose of sulphamethazin๕ $(8 \mathrm{mg} / \mathrm{kg})$ and the feasibility of carrying out th\& assay in a shorter time. Also, the frequency of the allele controlling slow acetylation, which is estimate 9 at $\mathrm{q}=0 \cdot 72 \pm 0 \cdot 3$, is similar to values which haver been found in Kenya, ${ }^{14}$ Uganda,${ }^{14}$ and norther Nigeria. ${ }^{8}$

\section{References}

1 Evans DAP, White TA. Human acetylation polymorphism J Lab Clin Med 1964;63:394-403.

2 Peters JH, Miller KS, Brown P. Studies on the metabolic bas for the genetically determined capacities for isoniazid in man J Pharmacol Exp Ther 1965;150:298-304.

3 Jeyakumar HL, French MR. Polymorphic acetylation of sulpha methazine in a Nigerian (Yoruba) population. Xenobiotic 1981;11:319-21.

+ Sunahara S, Urano M, Ogawa M. Genetical and geographic@ studies on isoniazid inactivation. Science 1961:134:1530-1. N

'White TA, Evans DAP. The acetylation of sulphamethazine and sulphamethoxypyridazine by human subjects. Clin PhaCo macol Ther 1968;9:80-5.

${ }^{\circ}$ Peters JH. Genetic factors in relation to drugs. Ann Rev Pharmacol 1968;8:427-48.

7 Ellard GA. Variations between individuals and populations the acetylation of isoniazid and its significance for the treatment of pulmonary tuberculosis. Clin Pharmacol Ther 1976;19 $610-25$.

${ }^{8}$ Fawcett IW, Gammon PT. Determination of the acetylate phenotype in a Northern Nigerian population. Tubercle 1975;5 199-201. 9 Eze IC, Obidoa O. Acetylation of sulphamethazine in $\frac{0}{2}$
Nigerian population. Biochem Genet 1978;16:1073-7.

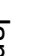


11) Varley H. Practical clinical biochemistry. 4th ed. London: Heinemann, 1962.

"Evans DAP. An improved and simplified method of detecting the acetylator phenotype. J Med Genet 1969:6:405-7.

12 Rao KEN, Michison DA. Nair KP. Tripathy SP. Sulphamethazine acetylation test for classification of patients as slow or rapid inactivators of isoniazid. $\mathrm{Br}$ Med $J$ 1970;ii:495-7.

13 Devadatta S. Gangdharam PRJ. Andrews RH, et al. Peripheral ncuritis due to isoniazid. Bull WHO 1960;23:587-90.
14 Ellard GA, Gammon PT, Tiitinen H. Determination of the acetylator phenotype using isoniazid. Tubercle 1975;56:203-9.

Correspondence and requests for reprints to Dr C F B Nhachi, Department of Clinical Pharmacology, Godfrey Huggins School of Medicine, PO Box A178, Avondale, Harare, Zimbabwe. 